Published in final edited form as:

Clin Lipidol. 2013 December ; 8(6): 659-667. doi:10.2217/clp.13.67.

\title{
Lipid signals and insulin resistance
}

\author{
Chongben Zhang ${ }^{1}$, Eric L Klett ${ }^{2}$, and Rosalind A Coleman ${ }^{1,}{ }^{*}$ \\ ${ }^{1}$ Department of Nutrition, University of North Carolina, Chapel Hill, NC 27599, USA \\ ${ }^{2}$ Department of Medicine, University of North Carolina, Chapel Hill, NC 27599, USA
}

\begin{abstract}
The metabolic syndrome, a cluster of metabolic derangements that include obesity, glucose intolerance, dyslipidemia and hypertension, is a major risk factor for cardiovascular disease. Insulin resistance has been proposed to be the common feature that links obesity to the metabolic syndrome, but the mechanism remains obscure. Although the excess content of triacylglycerol in muscle and liver is highly associated with insulin resistance in these tissues, triacylglycerol itself is not causal but merely a marker. Thus, attention has turned to the accumulation of cellular lipids known to have signaling roles. This review will discuss recent progress in understanding how glycerolipids and related lipid intermediates may impair insulin signaling.
\end{abstract}

\section{Keywords}

acyl-CoA; ceramide; diacylglycerol; lysophosphatidic acid; phosphatidic acid

\section{Obesity, lipids \& insulin resistance}

By 2020, more than 300 million people worldwide will be affected by the cardiometabolic risk factors that constitute the metabolic syndrome, obesity, glucose intolerance, dyslipidemia and hypertension [1,2]. The most accepted unifying theory to explain the pathophysiology of the metabolic syndrome is the presence of insulin resistance [3]. In insulin resistance, insulin becomes less effective at lowering blood glucose through diminished glucose uptake in peripheral tissues (muscle and adipose) and failure to suppress hepatic gluconeogenesis [4,5].

Hypotheses have been proposed to explain the molecular mechanism of insulin resistance including:

- A mismatch between fatty acid oxidation and the tricarboxylic acid cycle $[6,7]$;

- Inflammatory cytokines that cause endoplasmic reticulum (ER) stress and mitochondrial dysfunction [8];

- The production of signaling lipids, including ceramide, acyl-coenzyme As (acylCoAs), diacylglycerol (DAG) and phosphatidic acid (PA) $[9,10]$.

\footnotetext{
(C) 2013 Future Medicine Ltd

*Author for correspondence: Tel.: +1 919966 7213, Fax: +1 919843 8555, rcoleman@ unc.edu.

For reprint orders, please contact: reprints@futuremedicine.com
}

Financial \& competing interests disclosure

This work was supported by grants from the NIH DK090141 (to EL Klett) and DK59935 and DK56598 (to RA Coleman). The authors have no other relevant affiliations or financial involvement with any organization or entity with a financial interest in or financial conflict with the subject matter or materials discussed in the manuscript apart from those disclosed.

No writing assistance was utilized in the production of this manuscript. 
In this review, we will focus on recent studies of lipid intermediates related to, or derived from, triacylglycerol (TAG) biosynthesis and the mechanisms by which these lipids are thought to impair insulin signaling.

\section{TAG synthesis \& its association with insulin resistance}

Insulin resistance is strongly associated with excess TAG stores in multiple tissues, suggesting that insulin signaling might be diminished by the accumulation of specific lipids [11]. TAG biosynthesis begins with the activation of long-chain fatty acids by members of a family of acyl-CoA synthetases. Their long-chain acyl-CoA products are then esterified to glycerol-3-phosphate by glycerol-3-phosphate acyltransferase (GPAT) to form lysophosphatidic acid (LPA) [12]. This initial esterification step, catalyzed by four GPAT isoforms, is the rate-limiting step in TAG synthesis [12]. A second long-chain acyl-CoA is esterified to the resultant LPA product by one of at least three isoforms of acyl-GPAT (AGPAT; also called lysophosphatidic acid acyltransferase) to form PA, which is then hydrolyzed by one of three isoforms of PA phosphohydrolase (lipin) to form DAG. One of two isoforms of DAG acyltransferase esterifies the DAG with a third long-chain acyl-CoA to produce TAG.

Many studies have demonstrated the association between lipid accumulation and insulin resistance; however, as a neutral lipid sequestered in lipid droplets, TAG itself is unlikely to be a signaling molecule. Instead, an increasing body of evidence suggests that one or more of the lipid intermediates produced during TAG synthesis interfere with the intracellular insulin signaling pathway and contribute to the development of insulin resistance (Figure 1).

\section{Lysophosphatidic acid}

Indirect evidence that elevated LPA causes insulin resistance comes from rodent models in which GPAT was genetically manipulated. When GPAT1 was overexpressed in rat liver, LPA content increased approximately fourfold and a hyperinsulinemic-euglycemic clamp study demonstrated hepatic insulin resistance [13]. Conversely, when mice lacking GPAT1 $\left(\right.$ Gpat $\left.^{-/}\right)$were fed a high-fat diet, hepatic LPA content was reduced and the mice retained hepatic insulin sensitivity [14]. In addition to its production by de novo synthesis, LPA can also be generated by the hydrolysis of lysophosphatidylcholine by lysophospholipase D [15]. However, LPA generated from phospholipids has not been linked to insulin signaling.

In addition to effects mediated by plasma membrane-associated LPA receptors, LPA synthesized by GPAT1 can act as a ligand for the nuclear receptor PPAR $\gamma[16,17]$, whose activation improves insulin sensitivity in rodents and humans [18]. Since the overexpression of hepatic GPAT1 causes rats to become insulin resistant [13], it is unlikely that LPA derived from the de novo synthesis pathway activates PPAR $\gamma$. However, LPA derived from other pathways could activate PPAR $\gamma$ and lead to improved insulin sensitivity.

\section{Phosphatidic acid}

PA is produced by several pathways, including the hydrolysis of membrane phospholipids by phospholipase D (PLD), the phosphorylation of DAG by DAG kinase (DGK) and the esterification of LPA by AGPAT (Figure 1). The first evidence to suggest that PA regulates insulin sensitivity came from a study in which PLD-mediated production of PA activated the mTORC1 protein complex and its downstream targets [19]. When exogenous PA was added to cultured HEK293 cells, S6 kinase (S6K)-1 was activated in an mTORC1-dependent manner, and mTORC1 activation by serum was dependent on PLD1 [19]. Since the activation of mTORC1 and S6K1 enhances IRS-1 phosphorylation on Ser307, 632 and 636/639 residues [20,21], PA derived via PLD could cause insulin resistance by stimulating 
mTORC1-S6K1 signaling to increase serine phosphorylation of IRS-1. In contrast to the inhibition of insulin signaling by mTORC1, mTORC2 [22] enhances insulin signaling by phosphorylating and activating Akt at Ser473 [23]. Surprisingly, the PA derived via PLD action stabilizes both mTORC1 and mTORC2 complexes [24]. This stabilization suggests paradoxical outcomes with both enhanced and inhibited insulin signaling. However, the hypothesized effect of PLD-derived PA in regulating insulin signaling has not been directly investigated, and studies that relate PA content or PA treatment and mTOR activity are primarily associative; direct in vivo evidence for altered insulin signaling is lacking.

A second pathway that produces PA is the phosphorylation of DAG by one of ten DGK isoforms [25]. DGK manipulates the cellular content of DAG and PA simultaneously by phosphorylating DAG to form PA [26]. Although one study showed an association between DGK $\delta$ inhibition and insulin resistance in skeletal muscle [27], this study examined changes in DAG, but not PA. It is not known whether a DGK-mediated increase in PA content leads to impaired insulin signaling.

The third pathway of PA generation is the esterification of LPA by AGPAT. We have shown that overexpressing GPAT1 or AGPAT2 blocks insulin's ability to suppress hepatic glucose production [28]. In our study, overexpressing GPAT1 or AGPAT2 increased the cellular content of 16:0-containing PA approximately 2.2-fold. Of the various LPA, DAG and PA species that were tested, only di-16:0 PA disassociated the mTOR-rictor complex and inhibited mTORC 2 activity. Our results showed that in hepatocytes that overexpressed GPAT1, the absent phosphorylation of Akt at Ser473 and Thr308 diminished insulin signaling. This study provided direct evidence that a particular PA species inhibits insulin signaling.

PA appears to stabilize mTORC1 by interacting with the FKBP12-rapamycin-binding domain of mTOR [19]. PA has also been reported to activate and stabilize mTORC2 [24], probably by the same mechanism. These stabilization results focused on PA derived from the hydrolysis of membrane phospholipids by PLD. By contrast, PA produced via the glycerolipid synthetic pathway disrupts the mTORC2 complex [28]. It is unclear how PA originating from different sources can engender opposite effects on mTOR complex integrity. PA derived from both glycerolipid synthesis and from PLD-mediated phospholipid hydrolysis should be able to interact with the same FKBP12-rapamycin-binding domain of mTOR. Opposite effects on function may be due to each pathway of PA producing species that vary in their fatty acid composition. Although it is not known which PA species predominate in the PLD-derived PA that interacts with mTORC1 and mTORC2, the molecular species probably depend on the agent used to activate PLD, the cell type employed, and the phospholipid species that is hydrolyzed [29-32]. In contrast to the PA produced by overexpressing GPAT1, PLD-derived PA species are unlikely to contain predominately 16:0 fatty acids. Characterizing the nature of the pools of PA derived from PLD and from TAG synthesis will be critical to understanding normal and dysfunctional nutrient lipid signaling, particularly as it relates to nutrient surfeit and insulin resistance.

Although di-16:0 PA derived from GPAT1 overexpression inhibits mTORC2 in vitro [28], other PA species may inhibit mTORC2 activity in vivo. As it should be more difficult to dissociate an already formed mTORC2 complex than to prevent the initial association of the complex, lower concentrations of PA or different species might be effective in vivo.

\section{Diacylglycerol}

PKC $\varepsilon$ is thought to block the insulin signaling pathway by phosphorylating IRS1 at multiple serine residues [33], either directly or via activation of IKK $\beta$ and JNK [34-36]. Since DAG 
activates $\mathrm{PKC} \varepsilon$, hepatic DAG content is believed to link TAG synthesis and insulin sensitivity, particularly under conditions of hepatic steatosis [10]. Evidence exists both in favor of and against DAG as a major regulator of insulin signaling. Favoring DAG regulation are studies in which changes in GPAT alter DAG production. When GPAT1 is overexpressed in rat liver, insulin resistance occurs, DAG content increases twofold and $\mathrm{PKC} \varepsilon$ protein translocates from the cytosol to the membrane [13]. Conversely, in Gpat1 ${ }^{-/-}$ mice fed a high-fat diet, normal hepatic insulin sensitivity is retained, the hepatic DAG content is 2.5-fold lower than in controls fed a low-fat diet and the ratio of membrane- to

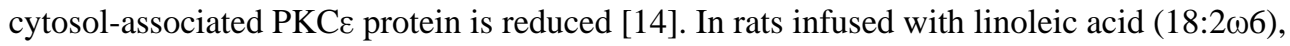
muscle becomes insulin resistant and soleus muscle DAG content increases, together with increased membrane localization of the $\mathrm{PKC} \varepsilon$ and $\mathrm{PKC} \theta$ proteins [34]. As with rats, humans infused with a $20 \%$ TAG emulsion (10\% safflower and $10 \%$ soybean oil) develop increased skeletal muscle DAG content and PKC ( $\beta$ and $\delta$ ) activity [37]. Feeding rats a 59\%-fat diet for 3 days causes hepatic insulin resistance [38], which is prevented by antisense oligonucleotide-mediated $P K C \varepsilon$ gene knockdown [33]. Although these data demonstrate a strong association between DAG, activated PKC isoforms and insulin resistance, most of these studies have reported changes only in the ratio of membrane- and cytosol-associated PKC isoforms as a proxy for PKC activation, and have not measured PKC activity directly.

Several human studies contradict the idea that excess DAG causes insulin resistance. For example, a 7-h intralipid infusion reduces insulin sensitivity in skeletal muscle (vastus lateralis), without changing the content of intramyocellular DAG [39]. Obese women are more insulin sensitive than obese men during saline or lipid infusion, without differences in vastus lateralis DAG concentration [40]. Although diabetic and nondiabetic subjects have similar intramyocellular DAG content, the diabetic patients have higher TAG content [41]. Similarly, the intramuscular DAG content is comparable in obese subjects with or without impaired glucose tolerance [42]. In normal-weight, endurance-trained athletes, the myocellular DAG content is twofold higher than in obese, sedentary subjects, but insulin sensitivity in the latter group is lower [43]. In addition, the muscle DAG content does not differ in lean insulin-sensitive subjects and obese people with insulin resistance [44].

Cell and animal studies also show that high DAG is not invariably accompanied by insulin resistance. In human primary myotubes and in mouse skeletal muscle, administration of ethyl-2-(6-[4-chlorophenoxy]hexyl)-oxirane-2-carboxylate (etomoxir), an inhibitor of carnitine palmitoyltransferase and fatty acid oxidation, results in improved insulin sensitivity, despite increased DAG content [45]. Mice deficient in hepatic microsomal TAG transfer protein have impaired VLDL secretion with hepatic steatosis and high DAG content, but retain normal insulin sensitivity [46]. Finally, liver-specific overexpression of DAG acyltransferase in mice results in increased hepatic TAG and DAG content, but, strikingly, no change in hepatic insulin sensitivity $[47,48]$.

These discrepancies in DAG-mediated insulin resistance may be due to the presence of distinct DAG species with differing signaling properties [49]. Alternatively, under some circumstances, the DAG might be sequestered in a nonsignaling pool (e.g., in lipid droplets) and, thus, not be able to activate PKC.

Cellular DAG content is also regulated by the activity of DGK (Figure 1). In patients with Type 2 diabetes, the skeletal muscle content of DGK $\delta$ protein and DGK activity is reduced [27]. In addition, in hyperglycemic, insulin-resistant Goto Kakizaki rats, reductions in DGK $\delta$ protein and DGK activity are accompanied by an increased content of DAG in the muscle [27]. The relationship of other DGK isoforms to insulin resistance has not been reported. 
When GPAT1 or AGPAT2 is overexpressed in mouse hepatocytes, the content of DAG species containing palmitate increases 3.4- and 1.7-fold, respectively, and correlates with the inhibition of mTORC2 activity and the phosphorylation of Akt at Ser473 and Thr308, and of GSK3a phosphorylation at Ser21. This correlation between cellular DAG content and mTORC2 activity and downstream signaling suggests that DAG synthesized de novo via the Kennedy pathway might contribute to hepatic insulin resistance [28]. Although the several DAG species evaluated did not disrupt the mTOR-rictor complex in vitro [28], hydrophobic DAG molecules may not be able to access the mTORC2 complex in vitro, which may not reflect in vivo conditions.

\section{Acyl-CoA \& ceramide}

Other lipids frequently cited as blunting insulin signaling are acyl-CoAs and ceramide. Long-chain acyl-CoA synthetases activate long-chain fatty acids to form acyl-CoAs, whose metabolic fates include entrance into pathways of complex lipid synthesis or mitochondrial $\beta$-oxidation [50,51]. Compared to animals on a control diet, rats fed a high-saturated-fat diet had 18 and $46 \%$ higher total acyl-CoA concentrations in liver and skeletal muscle, respectively [52]. Similarly, insulin resistance developed together with high skeletal muscle content of acyl-CoAs in rats fed a 59\%-safflower oil diet [53,54] or a 45\%-lard diet [55]. These studies provide evidence that long-chain acyl-CoAs may mediate the development of insulin resistance, either indirectly by increasing DAG, as described above, or by increasing ceramide, which inhibits hexokinase and diminishes glucose uptake, and which also activates $\mathrm{PKC} \theta$ to disrupt the insulin signaling pathway [56,57].

Other studies have reported that intracellular acyl-CoAs accumulate independent of insulin resistance. For example, mice deficient in either malonyl-CoA decarboxylase [57] or thioesterase superfamily member 1 [58] are protected from diet-induced insulin resistance, despite an elevated tissue content of acyl-CoA. Similarly, a deficiency of GPAT1 results in a 1.6-fold increase in the liver content of acyl-CoA, but ppat1 $^{-/-}$mice are protected from hepatic insulin resistance induced by a high-safflower oil diet [14]. Taken together, the studies relating elevated tissue acyl-CoA content and impaired insulin signaling remain inconclusive.

The evidence that ceramide reduces insulin signaling is better supported [59]. Depending upon the cell type, ceramide interferes with insulin signaling by two separate mechanisms, both of which involve Akt translocation to the plasma membrane. In $\mathrm{C} 2 \mathrm{C} 12$ myotubes, ceramide interferes with insulin signaling by enhancing the dephosphorylation of Akt by PP2A, thereby blocking the movement of Akt to the plasma membrane [60]. In rodent and human adipocytes, as well as L6 myotubes, ceramide inhibits the translocation of Akt to the plasma membrane by a mechanism involving the atypical PKC aPKC [61]. Ceramideactivated aPKC $\zeta$ phosphorylates Akt at Thr34 [61,62], leading to retention of the phosphorAkt in caveolin-enriched membranes [63] where it is unresponsive to insulin. It is unclear why there are differences between the two mechanisms and which mechanism prevails in vivo. Despite these differences, the common end point of ceramide-disrupted insulin signaling remains reduced active pAkt at the plasma membrane. In addition to these in vitro models, evidence from in vivo models supports the role of ceramide in insulin resistance. Using mice deficient in Toll-like receptor-4, researchers demonstrated that inflammation is also involved in ceramide-induced insulin resistance [64]. Although plasma ceramide is high in adolescents with Type 2 diabetes, it is not known whether the ceramide elevation is a cause or a consequence of diabetes [65]. Consistent with the idea that ceramide mediates insulin resistance, mice deficient in dihydroceramide desaturase 1, the enzyme that converts dihydroceramide into ceramide, retain insulin sensitivity after treatment with dexamethasone. This model is problematic, however, because the null animals have 
additional systemic problems and die by the age of 10 weeks [66]. More conclusive evidence for the role of ceramide in insulin resistance comes from mice fed a $60 \%$-fat diet [67]. Both the insulin resistance and doubling of skeletal muscle ceramide that developed by 12 weeks were blocked when myriocin, an inhibitor of ceramide synthesis, was fed concomitantly.

\section{Subcellular location and accessibility of lipid intermediates}

Since the enzymes involved in lipid metabolism are located on or within specific subcellular organelles, their lipid products vary in concentration in different subcellular compartments. Cellular compartmentalization of lipids may contribute to the ability of a lipid species to regulate insulin signaling. For example, different GPAT, AGPAT and DGK isoforms are located on multiple organelles [12]. PA is water-soluble and should be able to move within the cytoplasm, so its abundance in different compartments may vary depending on its site of origin. The PA produced by AGPAT isoforms located at the mitochondrial outer membrane or the ER [68] may have access to specific downstream pathways that differ from those accessible to PA produced by PLD1, which is primarily present in the Golgi and the nucleus [69]. The PA originating from the phosphorylation of DAG by DGK may be located near the cytoplasm, ER or nucleus, depending on the location of the specific DGK isoform [70]. Some evidence exists for these differences in function. PA that is derived from the PLD pathway activates $\mathrm{mTORC} 1$ signaling and inhibits insulin signaling by enhancing IRS1 phosphorylation at serine sites [19,71], whereas PA synthesized by the GPAT-AGPAT pathway inhibits mTORC2 signaling but has no effect on mTORC1 [28]. Another possible explanation for these findings is that the targets of PA have different locations; the mTORraptor complex that defines mTORC1 has a non-nuclear location, whereas the mTOR-rictor complex (mTORC2) is present in both nuclear and non-nuclear locations [72].

As DAG is a hydrophobic molecule, the enzymes that catalyze its conversion to PA or TAG must associate with membranes. In T cells, for example, DGKa redistributes between the plasma membrane and cytosol, and developmentally in neurons, DGK $\zeta$ moves between the nucleus and cytoplasm [70]. In other cell types, DGK $\beta$ colocalizes with actin filaments, DGK $\gamma$ resides in the Golgi complex and DGKe localizes to the ER [70]. The different subcellular locations of the DGK isoforms suggest that not all DGK isoforms play an equal role in DAG catabolism and PA production.

\section{Conclusion}

Most of the evidence for effects of lipid intermediates on insulin signaling has been correlative, and few studies have carefully examined the effects of a lipid species that originates from different pathways or from different subcellular locations. DAG has been widely accepted as causing insulin resistance in liver and muscle, but the evidence is primarily associative. More direct evidence exists for PA and ceramide. It is likely that many apparent discrepancies will be resolved by examining the fatty acid composition of the PA, DAG and ceramide species that increase as insulin resistance develops. The fact that PLD-derived PA stabilizes mTORC2, whereas GPAT1-derived PA disassociates mTORC2 strongly suggests that the two PA pools differ in some major way. It will be important to analyze the source, composition and location of the lipid intermediates, and to learn the precise mechanisms by which these molecules act to impair insulin signaling.

\section{Future perspective}

To fully understand the roles of lipid intermediates in the development of insulin resistance, one must carefully identify the putative lipid species that seem physiologically plausible. Which fatty acid composition of a lipid species is most effective? Are there functional 
differences between lipids that originate from different pathways (e.g., PA derived from PLD, DGK or GPAT)? Does it matter where the lipid is produced or where its target effector protein is located? Finally, what are the mechanisms involved and how do specific lipid molecules interact with putative modulators of insulin signaling such as PKC and mTOR?

It is our belief that insulin resistance is the result of multiple modes of cellular organelle dysfunction, including those of mitochondria and ER. In addition, aberrant signals that originate from oxidative stress and inflammation-related cytokines may also contribute to the development of insulin resistance. The presence of lipid intermediates may initiate or exacerbate the development of insulin resistance if they are present either in excess, in atypical subcellular locations or with unusual access to the nodes of the insulin-signaling pathway.

\section{References}

Papers of special note have been highlighted as:

•• of considerable interest

1. Mokdad AH, Ford ES, Bowman BA, et al. Prevalence of obesity, diabetes, and obesity-related health risk factors, 2001. JAMA. 2003; 289:76-79. [PubMed: 12503980]

2. Reaven $\mathrm{G}$. The insulin resistance syndrome: definition and dietary approaches to treatment. Annu Rev Nutr. 2005; 25:391-406. [PubMed: 16011472]

3. Mikhail N. The metabolic syndrome: insulin resistance. Curr Hypertens Rep. 2009; 11:156-158. [PubMed: 19278606]

4. Muoio DM, Newgard C. Mechanisms of disease: molecular and metabolic mechanisms of insulin resistance and beta-cell failure in Type 2 diabetes. Nat Rev Mol Cell Biol. 2008; 9:193-205. [PubMed: 18200017]

5. Taubes G. Insulin resistance. Prosperity's plague. Science. 2009; 325:256-260. [PubMed: 19608888]

6. Muoio DM. Intramuscular triacylglycerol and insulin resistance: guilty as charged or wrongly accused? Biochim Biophys Acta. 2010; 1801(3):281-288. [PubMed: 19958841]

7. Schooneman MG, Vaz FM, Houten SM, Soeters M. Acylcarnitines: reflecting or inflicting insulin resistance? Diabetes. 2013; 62:1-8. [PubMed: 23258903]

8. Hotamisligil GS, Erbay E. Nutrient sensing and inflammation in metabolic diseases. Nat Rev Immunol. 2008; 8:923-934. [PubMed: 19029988]

9. Coen PM, Goodpaster B. Role of intramyocelluar lipids in human health. Trends Endocrinol Metab. 2012; 23:391-398. [PubMed: 22721584]

10. Jornayvaz FR, Shulman G. Diacylglycerol activation of protein kinase Cepsilon and hepatic insulin resistance. Cell Metab. 2012; 15:574-584. [PubMed: 22560210]

11. Savage DB, Petersen KF, Shulman G. Disordered lipid metabolism and the pathogenesis of insulin resistance. Physiol Rev. 2007; 87:507-520. [PubMed: 17429039]

12•. Coleman RA, Mashek D. Mammalian triacylglycerol metabolism: synthesis, lipolysis, and signaling. Chem Rev. 2011; 111:6359-6386. Comprehensively describes the relationship between lipid metabolism, both lipid synthesis and lipolysis, and insulin signaling transduction. [PubMed: 21627334]

13. Nagle CA, An J, Shiota M, et al. Hepatic overexpression of glycerol-sn-3-phosphate acyltransferase 1 in rats causes insulin resistance. J Biol Chem. 2007; 282:14807-14815. [PubMed: 17389595]

14••. Neschen S, Morino K, Hammond LE, et al. Prevention of hepatic steatosis and hepatic insulin resistance in mitochondrial acyl-CoA:glycerol-sn-3-phosphate acyltransferase 1 knockout mice. Cell Metab. 2005; 2:55-65. Provides evidence that glycerol-3-phosphate acyltransferase-derived lipid signals impact insulin signaling in the liver and contribute to hepatic insulin resistance. [PubMed: 16054099] 
15. Nakanaga K, Hama K, Aoki J. Autotaxin - an LPA producing enzyme with diverse functions. J Biochem. 2010; 148:13-24. [PubMed: 20495010]

16. McIntyre TM, Pontsler AV, Silva AR, et al. Identification of an intracellular receptor for lysophosphatidic acid (LPA): LPA is a transcellular PPARgamma agonist. Proc Natl Acad Sci USA. 2003; 100:131-136. [PubMed: 12502787]

17. Stapleton CM, Mashek DG, Wang S, et al. Lysophosphatidic acid activates peroxisome proliferator activated receptor-gamma in CHO cells that over-express glycerol 3-phosphate acyltransferase-1. PLoS ONE. 2011; 6:e18932. [PubMed: 21533082]

18. Nolan JJ, Ludvik B, Beerdsen P, Joyce M, Olefsky J. Improvement in glucose tolerance and insulin resistance in obese subjects treated with troglitazone. N Engl J Med. 1994; 331:1188-1193. [PubMed: 7935656]

19. Fang Y, Vilella-Bach M, Bachmann R, Flanigan A, Chen J. Phosphatidic acid-mediated mitogenic activation of mTOR signaling. Science. 2001; 294:1942-1945. [PubMed: 11729323]

20. Um SH, D’Alessio D, Thomas G. Nutrient overload, insulin resistance, and ribosomal protein S6 kinase 1, S6K1. Cell Metab. 2006; 3:393-402. [PubMed: 16753575]

21. Gulati P, Thomas G. Nutrient sensing in the mTOR/S6K1 signalling pathway. BioChem Soc Trans. 2007; 35:236-238. [PubMed: 17371247]

22. Loewith R, Jacinto E, Wullschleger S, et al. Two TOR complexes, only one of which is rapamycin sensitive, have distinct roles in cell growth control. Mol Cell. 2002; 10:457-468. [PubMed: 12408816]

23••. Sarbassov DD, Guertin DA, Ali SM, Sabatini D. Phosphorylation and regulation of Akt/PKB by the rictor-mTOR complex. Science. 2005; 307:1098-1101. Demonstrates that mTORC2 is a key upstream regulator of $\mathrm{PKB} / \mathrm{Akt}$, providing an important mechanistic clue that mTORC2 participates in the regulation of insulin signaling transduction. [PubMed: 15718470]

24. Toschi A, Lee E, Xu L, Garcia A, Gadir N, Foster D. Regulation of mTORC1 and mTORC2 complex assembly by phosphatidic acid: competition with rapamycin. Mol Cell Biol. 2009; 29:1411-1420. [PubMed: 19114562]

25. Shulga YV, Topham MK, Epand R. Regulation and functions of diacylglycerol kinases. Chem Rev. 2011; 111:6186-6208. [PubMed: 21800853]

26. Merida I, Avila-Flores A, Merino E. Diacylglycerol kinases: at the hub of cell signalling. Biochem J. 2008; 409:1-18. [PubMed: 18062770]

27. Chibalin AV, Leng Y, Vieira E, et al. Downregulation of diacylglycerol kinase delta contributes to hyperglycemia-induced insulin resistance. Cell. 2008; 132:375-386. [PubMed: 18267070]

28••. Zhang C, Wendel AA, Keogh MR, Harris TE, Chen J, Coleman R. Glycerolipid signals alter mTOR complex 2 (mTORC2) to diminish insulin signaling. Proc Natl Acad Sci USA. 2012; 109:1667-1672. Provides direct evidence that phosphatidic acid, but not diacylglycerol, inhibits insulin signaling in the liver and contributes to hepatic insulin resistance. In addition, the mechanism appears to be that phosphatidic acid, especially that containing 16:0, disrupts mTOR/ rictor assembling. [PubMed: 22307628]

29. Divecha N, Lander DJ, Scott TW, Irvine R. Molecular species analysis of 1,2-diacylglycerols and phosphatidic acid formed during bombesin stimulation of Swiss 3T3 cells. Biochim Biophys Acta. 1991; 1093:184-188. [PubMed: 1863598]

30. Lee C, Fisher SK, Agranoff BW, Hajra A. Quantitative analysis of molecular species of diacylglycerol and phosphatidate formed upon muscarinic receptor activation of human SK-N-SH neuroblastoma cells. J Biol Chem. 1991; 266:22837-22846. [PubMed: 1744076]

31. Beckman BS, Mallia C, Clejan S. Molecular species of phospholipids in a murine stem-cell line responsive to erythropoietin. Biochem J. 1996; 314(Pt 3):861-867. [PubMed: 8615781]

32. Pettitt TR, Martin A, Horton T, Liossis C, Lord JM, Wakelam M. Diacylglycerol and phosphatidate generated by phospholipases $\mathrm{C}$ and $\mathrm{D}$, respectively, have distinct fatty acid compositions and functions. Phospholipase D-derived diacylglycerol does not activate protein kinase C in porcine aortic endothelial cells. J Biol Chem. 1997; 272:17354-17359. [PubMed: 9211874] 
33. Samuel VT, Liu ZX, Wang A, et al. Inhibition of protein kinase Cepsilon prevents hepatic insulin resistance in nonalcoholic fatty liver disease. J Clin Invest. 2007; 117:739-745. [PubMed: 17318260]

34. Yu C, Chen Y, Cline GW, et al. Mechanism by which fatty acids inhibit insulin activation of insulin receptor substrate-1 (IRS-1)-associated phosphatidylinositol 3-kinase activity in muscle. J Biol Chem. 2002; 277:50230-50236. [PubMed: 12006582]

35. Samuel VT, Petersen KF, Shulman G. Lipid-induced insulin resistance: unravelling the mechanism. Lancet. 2010; 375:2267-2277. [PubMed: 20609972]

36. Werner ED, Lee J, Hansen L, Yuan M, Shoelson S. Insulin resistance due to phosphorylation of insulin receptor substrate-1 at serine 302. J Biol Chem. 2004; 279:35298-35305. [PubMed: 15199052]

37. Itani SI, Ruderman NB, Schmieder F, Boden G. Lipid-induced insulin resistance in human muscle is associated with changes in diacylglycerol, protein kinase C, and IkappaB-alpha. Diabetes. 2002; 51:2005-2011. [PubMed: 12086926]

38. Kraegen EW, Clark PW, Jenkins AB, Daley EA, Chisholm DJ, Storlien L. Development of muscle insulin resistance after liver insulin resistance in high-fat-fed rats. Diabetes. 1991; 40:1397-1403. [PubMed: 1936601]

39. Hoeg LD, Sjoberg KA, Jeppesen J, et al. Lipid-induced insulin resistance affects women less than men and is not accompanied by inflammation or impaired proximal insulin signaling. Diabetes. 2010; 60:64-73. [PubMed: 20956497]

40. Vistisen B, Hellgren LI, Vadset T, et al. Effect of gender on lipid-induced insulin resistance in obese subjects. Eur J Endocrinol. 2008; 158:61-68. [PubMed: 18166818]

41. Anastasiou CA, Kavouras SA, Lentzas Y, Gova A, Sidossis LS, Melidonis A. Diabetes mellitus is associated with increased intramyocellular triglyceride, but not diglyceride, content in obese humans. Metabolism. 2009; 58:1636-1642. [PubMed: 19615699]

42. Perreault L, Bergman BC, Hunerdosse DM, Eckel R. Altered intramuscular lipid metabolism relates to diminished insulin action in men, but not women, in progression to diabetes. Obesity (Silver Spring). 2010; 18:2093-2100. [PubMed: 20379150]

43••. Amati F, Dube JJ, Alvarez-Carnero E, et al. Skeletal muscle triglycerides, diacylglycerols, and ceramides in insulin resistance: another paradox in endurance-trained athletes? Diabetes. 2011; 60:2588-2597. Suggests that diacylglycerol is not invariably linked to insulin resistance. [PubMed: 21873552]

44••. Coen PM, Hames KC, Leachman EM, et al. Reduced skeletal muscle oxidative capacity and elevated ceramide but not diacylglycerol content in severe obesity. Obesity (Silver Spring). 2013 (Epub ahead of print). Reviews discrepancies between the widely accepted theories of insulin resistance. Proposes that lipids, endoplasmic reticulum stress, inflammation and mitochondrial dysfunction are not, individually, the sole cause of insulin resistance, but instead, that multiple factors combine to produce insulin resistance. 10.1002/oby.20381

45. Timmers S, Nabben M, Bosma M, et al. Augmenting muscle diacylglycerol and triacylglycerol content by blocking fatty acid oxidation does not impede insulin sensitivity. Proc Natl Acad Sci USA. 2012; 109:11711-11716. [PubMed: 22753483]

46. Minehira K, Young SG, Villanueva CJ, et al. Blocking VLDL secretion causes hepatic steatosis but does not affect peripheral lipid stores or insulin sensitivity in mice. J Lipid Res. 2008; 49:2038-2044. [PubMed: 18515909]

47. Monetti M, Levin MC, Watt MJ, et al. Dissociation of hepatic steatosis and insulin resistance in mice overexpressing DGAT in the liver. Cell Metab. 2007; 6:69-78. [PubMed: 17618857]

48. Monetti M, Levin MC, Watt MJ, et al. Hepatic acyl-CoA:diacylglcyerol acyltransferase (DGAT) overexpression, diacylglycerol, and insulin sensitivity. Proc Natl Acad Sci USA. 2011; 108:e523. author reply: e524. [PubMed: 21807991]

49. Farese RV Jr, Zechner R, Newgard CB, Walther T. The problem of establishing relationships between hepatic steatosis and hepatic insulin resistance. Cell Metab. 2012; 15:570-573. [PubMed: 22560209]

50. Li LO, Klett EL, Coleman R. Acyl-CoA synthesis, lipid metabolism and lipotoxicity. Biochim Biophys Acta. 2009; 1801(3):246-251. [PubMed: 19818872] 
51. Soupene E, Kuypers F. Mammalian long-chain acyl-CoA synthetases. Exp Biol Med (Maywood). 2008; 233:507-521. [PubMed: 18375835]

52. Chen MT, Kaufman LN, Spennetta T, Shrago E. Effects of high fat-feeding to rats on the interrelationship of body weight, plasma insulin, and fatty acyl-coenzyme A esters in liver and skeletal muscle. Metabolism. 1992; 41:564-569. [PubMed: 1588840]

53. Oakes ND, Cooney GJ, Camilleri S, Chisholm DJ, Kraegen E. Mechanisms of liver and muscle insulin resistance induced by chronic high-fat feeding. Diabetes. 1997; 46:1768-1774. [PubMed: 9356024]

54. Ellis BA, Poynten A, Lowy AJ, et al. Long-chain acyl-CoA esters as indicators of lipid metabolism and insulin sensitivity in rat and human muscle. Am J Physiol Endocrinol Metab. 2000; 279:e554e560. [PubMed: 10950822]

55. Wright LE, Brandon AE, Hoy AJ, et al. Amelioration of lipid-induced insulin resistance in rat skeletal muscle by overexpression of Pgc-1beta involves reductions in long-chain acyl-CoA levels and oxidative stress. Diabetologia. 2011; 54:1417-1426. [PubMed: 21331471]

56. McGarry J. Banting lecture 2001: dysregulation of fatty acid metabolism in the etiology of Type 2 diabetes. Diabetes. 2002; 51:7-18. [PubMed: 11756317]

57. Koves TR, Ussher JR, Noland RC, et al. Mitochondrial overload and incomplete fatty acid oxidation contribute to skeletal muscle insulin resistance. Cell Metab. 2008; 7:45-56. [PubMed: 18177724]

58. Zhang Y, Li Y, Niepel MW, et al. Targeted deletion of thioesterase superfamily member 1 promotes energy expenditure and protects against obesity and insulin resistance. Proc Natl Acad Sci USA. 2012; 109:5417-5422. [PubMed: 22427358]

59. Chavez JA, Summers S. A ceramide-centric view of insulin resistance. Cell Metab. 2012; 15:585594. [PubMed: 22560211]

60. Cazzolli R, Carpenter L, Biden TJ, Schmitz-Peiffer C. A role for protein phosphatase 2A-like activity, but not atypical protein kinase Czeta, in the inhibition of protein kinase B/Akt and glycogen synthesis by palmitate. Diabetes. 2001; 50:2210-2218. [PubMed: 11574400]

61. Powell DJ, Hajduch E, Kular G, Hundal H. Ceramide disables 3-phosphoinositide binding to the pleckstrin homology domain of protein kinase B (PKB)/Akt by a PKCzeta-dependent mechanism. Mol Cell Biol. 2003; 23:7794-7808. [PubMed: 14560023]

62. Fox TE, Houck KL, O'Neill SM, et al. Ceramide recruits and activates protein kinase C zeta (PKC zeta) within structured membrane microdomains. J Biol Chem. 2007; 282:12450-12457. [PubMed: 17308302]

63. Hajduch E, Turban S, Le Liepvre X, et al. Targeting of PKCzeta and PKB to caveolin-enriched microdomains represents a crucial step underpinning the disruption in PKB-directed signalling by ceramide. Biochem J. 2008; 410:369-379. [PubMed: 17983354]

64. Holland WL, Bikman BT, Wang LP, et al. Lipid-induced insulin resistance mediated by the proinflammatory receptor TLR4 requires saturated fatty acid-induced ceramide biosynthesis in mice. J Clin Invest. 2011; 121:1858-1870. [PubMed: 21490391]

65. Lopez X, Goldfine AB, Holland WL, Gordillo R, Scherer P. Plasma ceramides are elevated in female children and adolescents with Type 2 diabetes. J Pediatr Endocrinol Metab. 2013; 26(910):995-998. [PubMed: 23612696]

66. Holland WL, Brozinick JT, Wang LP, et al. Inhibition of ceramide synthesis ameliorates glucocorticoid-, saturated-fat-, and obesity-induced insulin resistance. Cell Metab. 2007; 5:167179. [PubMed: 17339025]

67. Ussher JR, Koves TR, Cadete VJ, et al. Inhibition of de novo ceramide synthesis reverses dietinduced insulin resistance and enhances whole-body oxygen consumption. Diabetes. 2010; 59:2453-2464. [PubMed: 20522596]

68. Prasad SS, Garg A, Agarwal A. Enzymatic activities of the human AGPAT isoform 3 and isoform 5: localization of AGPAT5 to mitochondria. J Lipid Res. 2010; 52:451-462. [PubMed: 21173190]

69. Freyberg Z, Sweeney D, Siddhanta A, Bourgoin S, Frohman M, Shields D. Intracellular localization of phospholipase D1 in mammalian cells. Mol Biol Cell. 2001; 12:943-955. [PubMed: 11294898] 
70. Goto K, Hozumi Y, Nakano T, Saino-Saito S, Martelli A. Lipid messenger, diacylglycerol, and its regulator, diacylglycerol kinase, in cells, organs, and animals: history and perspective. Tohoku $\mathrm{J}$ Exp Med. 2008; 214:199-212. [PubMed: 18323690]

71. Zhang J, Gao Z, Yin J, Quon MJ, Ye J. S6K directly phosphorylates IRS-1 on Ser-270 to promote insulin resistance in response to TNF-(alpha) signaling through IKK2. J Biol Chem. 2008; 283:35375-35382. [PubMed: 18952604]

72. Rosner M, Hengstschlager M. Detection of cytoplasmic and nuclear functions of mTOR by fractionation. Methods Mol Biol. 2011; 821:105-124. [PubMed: 22125063] 


\section{Executive summary}

\section{Obesity, lipids \& insulin resistance}

- It has long been observed that obese people are predisposed to have insulin resistance, but the mechanism remains unclear. Lipid intermediates produced within the pathways of triacylglycerol (TAG) or sphingolipid synthesis have been associated with interrupted insulin signaling. Among these intermediates are diacylglycerol (DAG), which activates most forms of PKC, phosphatidic acid (PA), which alters mTOR kinase activity, and ceramides, which impair Akt signaling.

\section{TAG synthesis \& its association with insulin resistance}

- TAG itself probably does not directly inhibit insulin signaling; instead, lipid metabolites produced during the synthesis of TAG are likely to suppress insulin action.

\section{Lysophosphatidic acid}

- Elevated glycerol-3-phosphate acyltransferase-derived lysophosphatidic acid is associated with hepatic insulin resistance, but it remains uncertain whether the LPA has direct effects on insulin signaling.

\section{Phosphatidic acid}

- As a lipid messenger, PA may regulate insulin signaling via its interaction with mTORC1 and mTORC2. Direct evidence has shown that glycerol-3-phosphate acyltransferase-derived di-16:0 PA disrupts the association between mTOR and rictor.

\section{Diacylglycerol}

- DAG is widely accepted as a link between lipid metabolism and insulin resistance, due to its ability to activate conventional and novel PKC isoforms, which inhibit insulin signaling by phosphorylating serines of IRS1. However, some studies show an inconsistent relationship between DAG content and insulin resistance.

\section{Acyl-CoA \& ceramide}

- Although both long-chain acyl-CoAs and ceramides are believed to inhibit insulin signaling, the data for ceramides are more robust than those for longchain acyl-CoAs. Additional studies are needed to verify the effects of acyl-CoA in mediating lipid-associated insulin resistance.

\section{Subcellular location \& accessibility of lipid intermediates}

- As the enzymes involved in TAG and ceramide metabolism reside in multiple subcellular localizations, it is possible that the same lipid intermediate could exhibit different signaling functions depending on its origin. This may be particularly true for PA and DAG species that are produced by different pathways. 


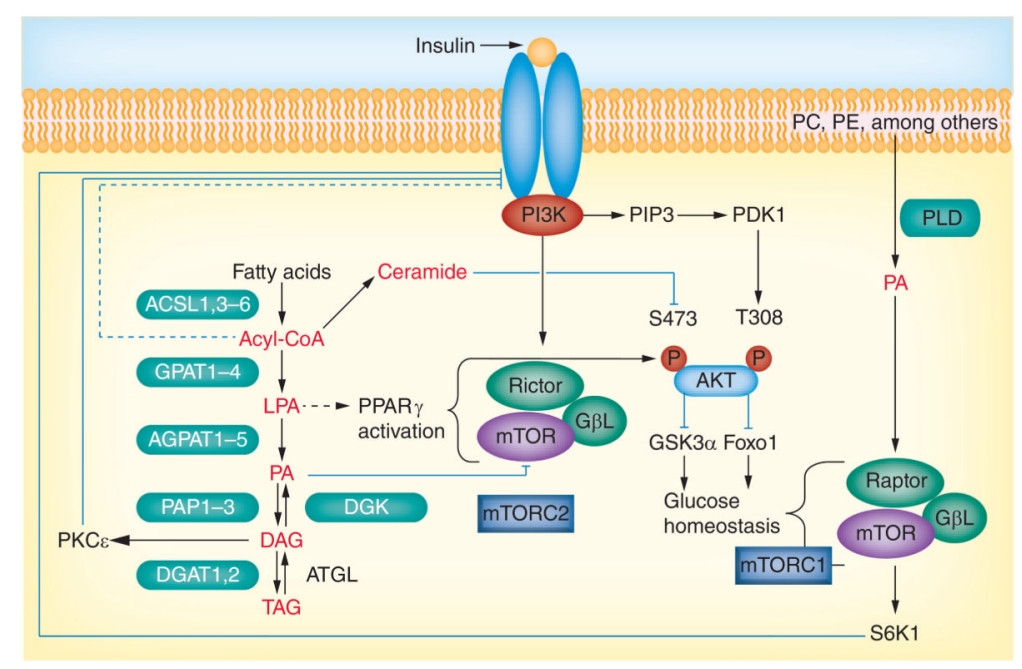

Figure 1. Triacylglycerol synthesis-derived lipid intermediates and insulin signaling regulation Long-chain fatty acids enter the cell and are activated by acyl-CoA synthetases to form acylCoAs, which are then esterified to glycerol-3-phosphate to form LPA by glycerol-3phosphate acyltransferase (GPAT). A second esterification by 1-acyl-GPAT produces PA. The phosphate is cleaved by PA phosphohydrolase (lipin) to form DAG and a third esterification converts the DAG to TAG. TAG is hydrolyzed by adipose TAG lipase to produce DAG, which can be phosphorylated by DAG kinase to form PA. Acyl-CoAs can also be converted to ceramide. Phospholipids can be hydrolyzed by PLD to form PA, LPA, PA, DAG, acyl-CoA and ceramides, which are all signaling molecules that may interfere with insulin signaling. PA originating from the GPAT pathway disrupts the mTOR-rictor complex to inhibit Akt phosphorylation and propagation of the insulin signal, and PA derived from PLD hydrolysis stabilizes mTOR/S6K1. DAG from the GPAT pathway activates conventional and novel PKC isoforms that phosphorylate IRS1 on serine residues and inhibit insulin signaling. LPA synthesized from the GPAT pathway may affect insulin signaling by activating PPAR $\gamma$. Acyl-CoAs may affect insulin signaling by interacting at the IRS1/PI3K node. Ceramides may affect glucose uptake by inhibiting hexokinase or may activate $\mathrm{PKC} \theta$, which disrupts insulin signaling. Dashed lines indicate that the interaction may be indirect and have intervening nodes.

Acyl-CoA: Acyl-coenzyme A; DAG: Diacylglycerol; DGK: DAG kinase; LPA: Lysophosphatidic acid; PA: Phosphatidic acid; PC: Phosphatidylcholine; PE: Phosphatidylethanolamine; PLD: Phospholipase D; TAG: Triacylglycerol. 